\title{
A religiosidade e a espiritualidade na atenção primária à saúde
}

\author{
Spirituality and religiosity in primary health care \\ La religiosidad y la espiritualidad en la atención primaria de la salud \\ Rodolfo Lima Araújo ${ }^{1 *}$, Antonio Luiz Gomes Junior ${ }^{1}$.
}

\begin{abstract}
RESUMO
Objetivo: Identificar e revisar a tendência das evidências científicas sobre a abordagem da religiosidade e espiritualidade na Atenção Primária à Saúde. Métodos: Revisão de literatura do tipo integrativa. $O$ levantamento foi realizado nas bases de dados da Literatura Latino-Americana e do Caribe de Ciências da Saúde (LILACS) e biblioteca eletrônica Scientific Electronic Library Online (SciELO). Os descritores utilizados foram: "Religiosidade" OR "Espiritualidade" AND "Atenção Primária à Saúde". Critérios de inclusão: artigos que contemplassem a temática, publicados nos idiomas português, inglês e espanhol no período de 2015 a 2020, foram excluídos artigos que não respondiam à questão norteadora, artigos que tinham como metodologia a revisão de literatura não sistemática, teses, dissertações e as publicações duplicadas. Resultados: A principal característica dos estudos foi: estudo transversal quantitativo, e com aumento não linear, ao longo do corte temporal. Parece que os aspectos religiosos são importantes para os indivíduos idosos, portadores de condições crônica e contra hábitos como fumar e ingerir bebida alcóolica. Em relação à saúde mental percebeu-se uma ambivalência. Considerações Finais: A abordagem sobre a religiosidade e espiritualidade deve ser abordada na Atenção Primária à Saúde, sendo esse desejo primariamente uma necessidade dos pacientes.
\end{abstract}

Palavras-chave: Espiritualidade, Religião, Atenção primária à saúde, Saúde da família, Assistência ao paciente.

\begin{abstract}
Objective: To identify and review the trend of scientific evidence on the approach of religiosity and spirituality in Primary Health Care. Methods: Review of integrative literature. The survey was conducted in the Latin American and Caribbean Health Sciences Literature (LILACS) and Scientific Electronic Library Online (SciELO) databases. The descriptors used were: "Religiosity" OR "Spirituality" AND "Primary Health Care". Inclusion criteria: articles that contemplated the theme, published in Portuguese, English and Spanish from 2015 to 2020, were excluded articles that did not answer the guiding question, articles that had as methodology the revision of non-systematic literature, theses, dissertations and duplicate publications. Results: The main characteristic of the studies was: quantitative cross-sectional study, and with non-linear increase, along the time section. It seems that religious aspects are important for elderly individuals, with chronic conditions and against habits such as smoking and drinking alcohol. In relation to the mental health, an ambivalence was noticed. Final Considerations: The approach about religiosity and spirituality must be approached in Primary Health Care, being this desire primarily a necessity of the patients.
\end{abstract}

Keywords: Spirituality, Religion, Primary health care, Family health, Patient care.

${ }^{1}$ Centro Universitário UNINOVAFAPI, Teresina - PI.

*E-mail: rodolfolima18@hotmail.com

SUBMETIDO EM: 9/2020

ACEITO EM: 10/2020

PUBLICADO EM: 1/2021 


\section{RESUMEN}

Objetivo: Identificar y revisar la tendencia de la evidencia científica sobre el enfoque de la religiosidad y la espiritualidad en la Atención Primaria de Salud. Métodos: Revisión de la literatura integradora. La encuesta se llevó a cabo en las bases de datos de Literatura Latinoamericana y del Caribe en Ciencias de la Salud (LILACS) y de la Biblioteca Científica Electrónica en Línea (SciELO). Los descriptores utilizados fueron: "Religiosidad" O "Espiritualidad" Y "Atención Primaria de Salud". Criterios de inclusión: artículos que contemplaban el tema, publicados en portugués, inglés y español de 2015 a 2020, se excluyeron los artículos que no respondían a la pregunta orientadora, los artículos que tenían como metodología la revisión de literatura no sistemática, las tesis, las disertaciones y las publicaciones duplicadas. Resultados: La principal característica de los estudios fue: estudio cuantitativo transversal, y con aumento no lineal, a lo largo de la sección temporal. Parece que los aspectos religiosos son importantes para las personas mayores, con condiciones crónicas y contra hábitos como fumar y beber alcohol. En relación con la salud mental, se percibió una ambivalencia. Consideraciones finales: El enfoque sobre la religiosidad y la espiritualidad debe ser abordado en la Atención Primaria de Salud, siendo este deseo principalmente una necesidad de los pacientes.

Palabras clave: Espiritualidad, La religión, Atención primaria de salud, Salud familiar, Cuidado del paciente.

\section{INTRODUÇÃO}

A relação entre Religiosidade e Espiritualidade (R/E) e saúde, ainda permeia pelos campos do conflito e da amistosidade, ora por questionamentos de incompatibilidade da razão (ciência) com os aspectos da R/E, ora pelo descredito de incapacidade de avaliação científica destes. Todavia, essa discussão vem sendo cada vez mais diluída por estudos científicos robustos que demonstram interferência indireta e diretamente dos aspectos religiosos no bem-estar, considerando-os como determinantes da saúde (ALMEIDA AM e LUCHHETTI G, 2016).

Apesar de parecem, e até serem utilizados como semelhantes, os conceitos de religiosidade e espiritualidade são distintos. Entende-se por religiosidade as atividades desempenhadas pelos indivíduos relacionada às suas crenças, podendo ou não estar alicerçadas em uma determinada religião. Já o conceito de espiritualidade transcende os aspectos sagrados, este perfaz pela ideação de sentido da vida, da elevação pessoal, algo que por vezes, para alguns, ultrapassam a linguagem (VALENTE TCO, et al. ,2016).

A capacidade de enfrentamento de situações estressoras ou eventos adversos, através da R/E nos traz o conceito de coping religioso ou coping religioso espiritual (CRE). Diversos estudos, sobretudo mais recentemente, têm demonstrado impacto importante do CRE na morbimortalidade principalmente sobre a espera da saúde mental, isso representa o CRE positivo, porém, esse enfrentamento pode se insinuar prejudicial, acarretando danos à saúde a partir de crenças e convicções religiosas, sendo este um exemplo de CRE negativo (CORRÊA CV, et al., 2016).

Diversos fatores podem ser elencados para falta da abordagem sobre a $R / E$ na prática clínica como: limitação de tempo para consulta, medo na imposição de crenças pessoais, e até a pouca ou inexistente abordagem sobre a temática na formação acadêmica, isso contribui para não percepção dos valores religiosos e espirituais como determinantes de saúde (PANITZ GO, et al., 2018; INOUE TM e VERCINA MV, 2017).

Pensando em um modelo ampliado de saúde, com uma abordagem holística, biopsicossocial, centrada na pessoa, os aspectos espirituais devem ser abordados no campo do processo saúde-doença (DE FIGUEIRÊDO RAM, et al., 2020).

A abordagem dos diversos determinantes da saúde pode ser feita em todos os níveis de atenção, sobretudo na atenção primária à saúde (APS), que tem como um de seus atributos derivados a competência 
cultura, ou seja, deve-se levar em consideração a natureza intima de cada indivíduo, a singularidade com que cada pessoa se apresenta e se sente representada segundo seus valores, crenças, hábitos e costumes (DE FIGUEIRÊDO RAM, et al., 2020).

Pensando também sobre um foco de APS abrangente, a compreensão de saúde não deve ser dissociada dos determinantes sociais do processo saúde-doença, como a R/E (GIOVANELLA L, 2009). Portanto, tendo a atenção primária a função de porta de entrada para os serviços de saúde e, se bem articulada, tem grande poder de resolutividade dos problemas, os aspectos relacionados a religião, espiritualidade e religiosidade devem ser reconhecidos como determinantes importantes do processo saúde doença.

A presente revisão integrativa tem como objetivo identificar a tendência das evidências científicas sobre a abordagem da religiosidade e espiritualidade na APS, tanto sob os aspectos relacionados aos usuários quanto dos profissionais que atuam neste nível de atenção.

Tal investigação sustenta-se na inquietude em contribuir para uma visão holística, biopsicossocial e espiritual do processo saúde-doença, na necessidade dos pacientes de serem questionados sobre os aspectos da R/E pelos profissionais de saúde, bem como contribuir para o desenvolvimento de pesquisas vindouras que busquem compreender o indivíduo/usuário como um todo, sem desvinculá-lo de segmentos que potencialmente interferem na saúde.

\section{MÉTODOS}

Trata-se de uma revisão de literatura do tipo integrativa. Este método que possibilita uma ampla gama de informações sobre determinada problemática, constituindo uma forma grandiosa de conhecimento e rigor metodológico.

A síntese desta metodologia de pesquisa facilita a incorporação de evidências, o que permite uma praticidade de novo conhecimento para a prática clínica (DE SOUSA MM, 2017). A inquietação condutora dessa pesquisa é: qual a tendência das evidências científicas sobre a religiosidade e espiritualidade na Atenção Primária à Saúde?

O levantamento na literatura foi realizado nas bases de dados da Literatura Latino-Americana e do Caribe de Ciências da Saúde (LILACS) e biblioteca eletrônica Scientific Electronic Library Online (SciELO) para tal, utilizou-se da combinação de descritores controlados, cadastrados nos Descritores em Ciências da Saúde (DECS): "Religiosidade" OR "Espiritualidade" AND "Atenção Primária à Saúde".

Como critérios de inclusão foram estabelecidos: artigos científicos que contemplassem a temática, publicados nos idiomas português, inglês e espanhol no período correspondente de 2015 a 2020. A partir da combinação dos descritores foram obtidos 28 estudos.

Numa avaliação inicial por meio dos resumos, verificou-se que 03 destes estavam fora do recorte temporal, foram excluídos artigos que não respondiam à questão norteadora, artigos que tinham como metodologia a revisão de literatura não sistemática, teses, dissertações e as publicações duplicadas, portanto, 12 artigos foram excluídos.

Sendo possível selecionar 13 artigos para compor a amostra final desta revisão integrativa da literatura. A Figura 1 apresenta o fluxograma concernente ao processo de seleção dos artigos que compõem este estudo, em consonância com as bases de dados, critérios de inclusão e exclusão no período de 2015 a 2020. 
Figura 1 - Fluxograma de amostragem da revisão integrativa.

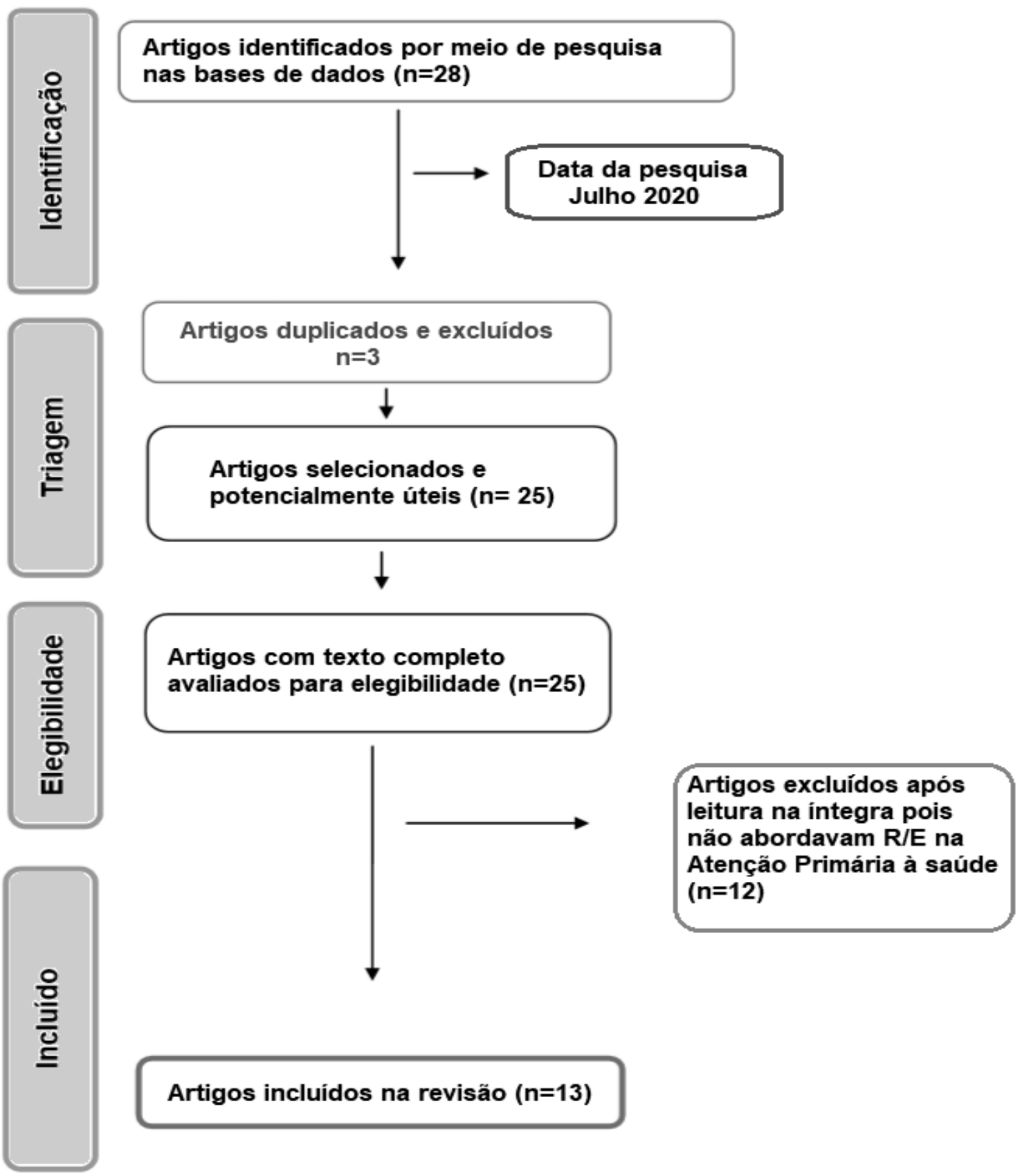

Fonte: Araujo RL e Junior ALG, 2020.

A análise dos dados foi realizada de forma descritiva. Os estudos foram reunidos em 2 grupos, os artigos que tratavam apenas a temática específica sobre a ótica do paciente ou usuário, outro grupo, artigos que trouxeram as experiências da R/E com os profissionais, o que permitiu avaliar os níveis de evidências, bem como identificar a necessidade de investigações futuras acerca da temática.

\section{RESULTADOS E DISCUSSÃO}

Nesta revisão integrativa, foram analisados 13 artigos que trouxeram investigação sobre a questão norteadora seguindo também os critérios de inclusão. Os artigos foram lidos na integra, analisados, caracterizados para posteriormente serem discutidos. As características dos artigos estão apresentadas no Quadro 1. 


\section{Revista Eletrôni3a Acervo Saúde / Electronic Journal Collection Health ISSN 2178-2091}

Quadro 1 - Fontes bibliográficas incluídas na revisão integrativa, segundo periódico/ano, título, autores, métodos e ideia central.

\begin{tabular}{|c|c|c|c|c|c|}
\hline $\begin{array}{l}\text { Periódico/ } \\
\text { Ano de } \\
\text { publicação }\end{array}$ & Título & Autores & Métodos & Ideia central & $\begin{array}{l}\text { Nível de } \\
\text { Evidência }\end{array}$ \\
\hline $\begin{array}{l}\text { Int J } \\
\text { SocPsychiatry/ } \\
2015\end{array}$ & $\begin{array}{l}\text { Entre os valores modernos e a tradição: } \\
\text { Atitudes informais de busca de ajuda à } \\
\text { saúde mental de acordo com as mulheres } \\
\text { israelenses, pacientes da atenção } \\
\text { primária e seus provedores. }\end{array}$ & $\begin{array}{l}\text { Ayalon L, et } \\
\text { al. (2015) }\end{array}$ & Estudo qualitativo & $\begin{array}{l}\text { As mulheres árabes israelenses subutilizam os } \\
\text { serviços de saúde mental. }\end{array}$ & 4 \\
\hline $\begin{array}{l}\text { Acta Paulista } \\
\text { de Enf./2016 }\end{array}$ & $\begin{array}{l}\text { Associação entre o consumo de bebidas } \\
\text { alcoólicas e tabaco e a religiosidade }\end{array}$ & $\begin{array}{l}\text { Queiroz } \\
\text { NR, et al. } \\
(2016)\end{array}$ & $\begin{array}{l}\text { Estudo } \\
\text { quantitativo } \\
\text { transversal. }\end{array}$ & $\begin{array}{l}\text { Verificar a associação entre a religiosidade e o } \\
\text { padrão de consumo de álcool e tabaco em } \\
\text { população atendida na Atenção Primária à Saúde. }\end{array}$ & 4 \\
\hline $\begin{array}{c}\text { Saúde em } \\
\text { Debate/2017 }\end{array}$ & $\begin{array}{l}\text { Idosos longevos: avaliação da qualidade } \\
\text { de vida no domínio da espiritualidade, da } \\
\text { religiosidade e de crenças pessoais. }\end{array}$ & $\begin{array}{l}\text { Nunes } \\
\text { MGS, et al. } \\
(2017)\end{array}$ & $\begin{array}{l}\text { Estudo descritivo, } \\
\text { quantitativo, de } \\
\text { corte transversal. }\end{array}$ & $\begin{array}{l}\text { Avaliar a qualidade de vida no domínio da } \\
\text { espiritualidade, da religiosidade, de crenças } \\
\text { pessoais e fatores associados em idosos longevos. }\end{array}$ & 4 \\
\hline $\begin{array}{l}\text { Revista } \\
\text { Brasileira de } \\
\text { Educação } \\
\text { Médica/2017 }\end{array}$ & $\begin{array}{c}\text { A Religiosidade/Espiritualidade dos } \\
\text { Médicos de Família: Avaliação de Alunos } \\
\text { da Universidade Aberta do SUS (UNA- } \\
\text { SUS). }\end{array}$ & $\begin{array}{l}\text { Aguiar PR, } \\
\text { et al. } \\
(2017)\end{array}$ & $\begin{array}{l}\text { Estudo descritivo, } \\
\text { quantitativo, de } \\
\text { corte transversal. }\end{array}$ & $\begin{array}{c}\text { Apresentar a opinião de médicos, especializando } \\
\text { em Saúde da sobre essa temática na prática clínica } \\
\text { e os índices de religiosidade/espiritualidade dos } \\
\text { estudantes. }\end{array}$ & 4 \\
\hline $\begin{array}{c}\text { J Relig } \\
\text { Health/2017 }\end{array}$ & $\begin{array}{l}\text { Prevalência do tabagismo entre os } \\
\text { usuários de unidades primárias de saúde } \\
\text { no Brasil: O Papel da Religiosidade. }\end{array}$ & $\begin{array}{l}\text { Martinez } \\
\text { EZ, et al. } \\
(2017)\end{array}$ & $\begin{array}{l}\text { Estudo descritivo, } \\
\text { quantitativo, de } \\
\text { corte transversal. }\end{array}$ & $\begin{array}{l}\text { Examinar a associação entre envolvimento } \\
\text { religioso e uso de tabaco em uma grande amostra } \\
\text { representativa de usuários de unidades básicas de } \\
\text { saúde de Ribeirão Preto, SP }\end{array}$ & 4 \\
\hline
\end{tabular}

REAS/EJCH | Vol.13(1) | e5418 | DOI: https://doi.org/10.25248/reas.e5418.2021 Página 5 de 11 
Revista Eletrôni3a Acervo Saúde / Electronic Journal Collection Health ｜ ISSN 2178-2091

\begin{tabular}{|c|c|c|c|c|c|}
\hline $\begin{array}{l}\text { Periódico/ } \\
\text { Ano de } \\
\text { publicação }\end{array}$ & Título & Autores & Métodos & Ideia central & $\begin{array}{l}\text { Nível de } \\
\text { Evidência }\end{array}$ \\
\hline $\begin{array}{l}\text { RevBrasMedF } \\
\text { am Com. } \\
\text { /2018 }\end{array}$ & $\begin{array}{l}\text { Espiritualidade e resiliência na atenção } \\
\text { domiciliar }\end{array}$ & $\begin{array}{l}\text { Zandavalli, } \\
\text { RB, et al. } \\
\text { (2018) }\end{array}$ & $\begin{array}{l}\text { estudo } \\
\text { quantitativo } \\
\text { transversal e } \\
\text { descritivo }\end{array}$ & $\begin{array}{l}\text { Avaliar a relação entre religiosidade e } \\
\text { espiritualidade com resiliência em pacientes em AD } \\
\text { de Unidades de Saúde de Atenção Primária à } \\
\text { Saúde }\end{array}$ & 4 \\
\hline $\begin{array}{l}\text { Rev. Bras. } \\
\text { Enferm/2018 }\end{array}$ & $\begin{array}{l}\text { Anciãos que cuidam dos idosos: } \\
\text { espiritualidade como alívio do estresse }\end{array}$ & $\begin{array}{l}\text { Da Silva } \\
\text { MCM, et al. } \\
(2018)\end{array}$ & $\begin{array}{l}\text { Estudo } \\
\text { qualitativo, com } \\
\text { aporte teórico- } \\
\text { metodológico da } \\
\text { GroundedTheory }\end{array}$ & $\begin{array}{l}\text { Investigar as formas de enfrentamento utilizadas } \\
\text { para alívio de tensões por cuidadores idosos de } \\
\text { familiares idosos e conhecer o tipo de apoio que } \\
\text { recebem do serviço de Atenção Primária à Saúde } \\
\text { no domicílio. }\end{array}$ & 4 \\
\hline $\begin{array}{l}\text { J Nurs UFPE } \\
\text { online/2018 }\end{array}$ & $\begin{array}{l}\text { Itinerários terapêuticos em sujeitos com } \\
\text { Diabetes Mellitus: linhas fortes e fracas. }\end{array}$ & $\begin{array}{l}\text { Coqueiro } \\
\text { JM, et al. } \\
(2018)\end{array}$ & $\begin{array}{l}\text { Trata-se de um } \\
\quad \text { estudo } \\
\text { documental de } \\
\text { abordagem quali } \\
\text { quantitativa }\end{array}$ & $\begin{array}{c}\text { Analisar, a partir do referencial teórico da análise } \\
\text { institucional, as matérias veiculadas sobre o DM } \\
\text { pela mídia impressa do estado do Espírito Santo, } \\
\text { Brasil. }\end{array}$ & 4 \\
\hline $\begin{array}{l}\text { Revista } \\
\text { Brasileira de } \\
\text { Cancerologia/ } \\
2018\end{array}$ & $\begin{array}{l}\text { Qualidade de Vida Relacionada à Saúde } \\
\text { e Espiritualidade em Pessoas com } \\
\text { Câncer }\end{array}$ & $\begin{array}{l}\text { Menezes } \\
\text { RM, et al. } \\
(2018)\end{array}$ & $\begin{array}{l}\text { Estudo } \\
\text { transversal } \\
\text { quantitativo. }\end{array}$ & $\begin{array}{l}\text { Caracterizar a qualidade de vida relacionada à } \\
\text { saúde - QVRS — de pacientes com câncer } \\
\text { avançado nas literaturas nacional e internacional }\end{array}$ & 4 \\
\hline
\end{tabular}

REAS/EJCH | Vol.13(1) | e5418 | DOI: https://doi.org/10.25248/reas.e5418.2021 Página 6 de 11 


\begin{tabular}{|c|c|c|c|c|c|}
\hline $\begin{array}{l}\text { Periódico/ } \\
\text { Ano de } \\
\text { publicação }\end{array}$ & Título & Autores & Métodos & Ideia central & $\begin{array}{l}\text { Nível de } \\
\text { Evidência }\end{array}$ \\
\hline $\begin{array}{c}\mathrm{J} \\
\text { ProfNurs/2018 }\end{array}$ & $\begin{array}{l}\text { Formação espiritual, secularização, e } \\
\text { reforma da enfermagem e educação } \\
\text { profissional na América }\end{array}$ & $\begin{array}{l}\text { Libster MM } \\
\text { (2018) }\end{array}$ & Estudo de Caso & $\begin{array}{l}\text { Apresentar uma análise histórica das crenças, } \\
\text { receitas e remédios para a saúde das três } \\
\text { comunidades, fundamentais para a formação e } \\
\text { educação espiritual dos profissionais de } \\
\text { enfermagem em suas comunidades. }\end{array}$ & 4 \\
\hline $\begin{array}{l}\text { Cadernos } \\
\text { Saúde } \\
\text { Coletiva/2019 }\end{array}$ & $\begin{array}{l}\text { Religiosidade e padrões de consumo de } \\
\text { álcool entre usuários de unidades de } \\
\text { atenção primária à saúde no Brasil }\end{array}$ & $\begin{array}{l}\text { Martinez } \\
\text { EZ, et al. } \\
(2019)\end{array}$ & $\begin{array}{l}\text { Estudo } \\
\text { transversal } \\
\text { quantitativo }\end{array}$ & $\begin{array}{l}\text { Examinar a associação entre envolvimento } \\
\text { religioso e padrões de consumo de álcool em } \\
\text { unidades de atenção primária à saúde de Ribeirão } \\
\text { Preto, Brasil. }\end{array}$ & 4 \\
\hline $\begin{array}{l}\text { Ciência \& } \\
\text { Saúde } \\
\text { Coletiva/2020 }\end{array}$ & $\begin{array}{l}\text { Idosos cuidadores de outros idosos que } \\
\text { residem com e sem crianças: sobrecarga, } \\
\text { otimismo e estratégias de enfrentamento }\end{array}$ & $\begin{array}{l}\text { Oliveira } \\
\text { NA, et al. } \\
(2020)\end{array}$ & $\begin{array}{l}\text { Estudo } \\
\text { transversal } \\
\text { quantitativo. }\end{array}$ & $\begin{array}{l}\text { Comparar o nível de sobrecarga, otimismo e as } \\
\text { estratégias de enfrentamento de idosos cuidadores } \\
\text { de idosos que residem com e sem crianças. }\end{array}$ & 4 \\
\hline $\begin{array}{l}\mathrm{J} \\
\text { InterprofCare/ } \\
2020\end{array}$ & $\begin{array}{c}\text { A orientação espiritual e religiosa afeta a } \\
\text { prática clínica dos prestadores de } \\
\text { serviços de saúde? }\end{array}$ & $\begin{array}{l}\text { Palmer KE, } \\
\text { et al. } \\
(2020)\end{array}$ & $\begin{array}{l}\text { Pesquisa } \\
\text { Descritiva } \\
\text { Transversal }\end{array}$ & $\begin{array}{c}\text { Avaliar as crenças e práticas religiosas e espirituais } \\
\text { entre os prestadores de serviços de saúde, }\end{array}$ & 4 \\
\hline
\end{tabular}

Fonte: Araujo RL e Junior ALG, 2020. 
Os estudos selecionados para esta pesquisa de revisão integrativa, tiveram como como características relacionada ao desenvolvimento no tempo, os estudos de corte transversal em sua totalidade, variando quanto a forma de abordagem, sendo a maioria correspondente a estudos do tipo quantitativo.

Esse fato pode demonstrar uma certa carência de estudos que avaliem um impacto mais observacional longitudinal dos aspectos inerentes à R/E na atenção primária à saúde, bem como possíveis relações deste determinante com patologias ou condições de saúde crônicas a longo prazo.

Com relação ao ano de publicação, percebemos uma maior quantidade de artigos no ano de 2018. É notório que o crescimento de publicações sobre a referida temática com o decorrer dos anos, não se apresentou de forma uniforme, todavia percebemos que o interesse pelo objeto, como questão importante para o processo saúde-doença, e de intriga entre os pesquisadores, vem crescendo, denotando a necessidade tanto de uma melhor abordagem dos aspectos relacionados à $\mathrm{R} / \mathrm{E}$ na prática diária em saúde, como novos estudos que corroborem para fundamentação desta evidência.

As dificuldades para reconhecer o papel da R/E como provável determinante de saúde se inicia desde a formação acadêmica ou técnica dos profissionais desta área. Pouco, ou muito superficialmente é inserido nos conteúdos programáticos curriculares e mesmo de extensão, essa visão do indivíduo holístico, do indivíduo como algo abrangente, sem desvincular este do meio social, cultural, além das poucas referências que abordem essa temática na APS que tem como um de seus atributos a integralidade do cuidado (AGUIAR PR, et al., 2017; QUEIROZ NR, et al., 2015).

O nível de atenção primário, tem como objetivo fazer uma prática clínica centrada na pessoa, levando em conta as singularidades e particularidades inerente a cada indivíduo. É nesta premissa que se insere a principal argumentação para a abordagem da espiritualidade e/ou religiosidade em qualquer cenário ou oportunidade.

Essa prática de abordagem holística, refere-se ao segundo componentedo método clínico centrado na pessoa, que procura o entendimento do indivíduo como um todo, provido de um contexto proximal (família) e distal (social) (AGUIAR PR, et al., 2017).

O próprio conceito de saúde, defendido pela Organização Mundial de Saúde (OMS), introduz além de outras dimensões como: biológica, social e mental, a espiritual como campo importante para o bem-estar. A necessidade da abordagem dos aspectos religiosos na saúde parte, primariamente, dos próprios indivíduos, do usuário dos serviços de saúde, que na maioria das vezes, consideram a religião como fator determinante para a qualidade de vida, e sentem falta desta abordagem pelos profissionais da saúde na sua lida diária (NUNES MGS, et al., 2017).

O que podemos perceber na realidade é uma verdadeira lacuna entre o desejo do usuário de ser abordado sobre os aspectos religiosos e a falta de questionamento sobre a temática, reforçando, portanto, a necessidade de reconhecimento e treinamento para os profissionais da APS sobre a abordagem da espiritualidade na prática.

Esse treinamento, pode se constituir numa ferramenta estratégica de sensibilização profissional, para acolher as demandas dos indivíduos que transcendem os aspectos biomédico das patologias, ou mesmo da importância de proporcionar a abordagem da $\mathrm{R} / \mathrm{E}$ como algo inerente à saúde e não somente à doença (NUNES MGS, et al., 2017; ZANDAVALLI RB, et al., 2019).

Diversos fatores são atribuídos na literatura para explicar a dificuldade na abordagem da espiritualidade na APS, mesmo que os profissionais deste nível de atenção reconheçam que R/E seja importante no processo saúde-doença.

São fatores citados com frequência que corroboram para essa dificuldade: a falta de tempo, uma vez que os profissionais lidam com demandas por vezes excessivas de atendimento; a falta de treinamento para aperfeiçoamento de habilidades que facilitem essa abordagem; o medo de imposição de valores pessoais relacionados a espiritualidade ou medo de ofensa ao paciente e as suas crenças (ZANDAVALLI RB, et al., 2019). 
Tendo em vista que o processo de transição demográfica e epidemiológica traz consigo uma maior preocupação com as condições crônicas de saúde e com o envelhecimento populacional, a atenção primaria à saúde, como porta de entrada para os serviços de saúde e responsável pelo cuidado longitudinal, deve estar preparada para atender as necessidade destes usuários longevos.

E é justamente neste público que se percebe a maior necessidade de abordagem sobre os aspectos religiosos, uma vez que é nesta faixa etária onde encontram-se maiores índices de religiosidade intrínseca, além de maior associação da espiritualidade como fator protetor para as adversidades (OLIVEIRA NA, et al. 2020; DA SILVA MC, et al., 2018).

$\mathrm{Na}$ faixa etária acima de 60 anos, o coping religioso e espiritual é bastante evidenciado como enfrentamento das problemáticas, das tensões emocionais e sobrecargas negativas. Outro fator atribuído à R/E neste grupo etário, é a possibilidade de melhor comunicação e estreitamento das relações familiares, além do desenvolvimento de hábitos que favoreçam o bem-estar, como também possíveis experiências de cura.

A abordagem dos aspectos religiosos para esses indivíduos pode ser feita de forma simples e rápida através, por exemplo, do questionário FICA (acrônimo para: fé, importância, comunidade e ação do tratamento). Caso exista a evidência que o idoso se considere não religioso, a abordagem deve ter como foco a espiritualidade, ou seja, algo que lhe dê sentido à vida, com intuito de investigar possíveis crenças atreladas ao processo saúde-doença (DA SILVA MC, et al., 2018).

Para os cuidadores de indivíduos idosos, a religiosidade aparece também como forte fator protetivo. Esses indivíduos que atuam no cuidado à população senil, geralmente são expostos a altas demandas estressoras como: sobrecarga de trabalho, dupla ou tripla jornada e dependências decorrentes das limitações físicas e cognitivas.

Todos estes fatores geradores de estresse precisam ser atenuados de forma a melhorar as condições de saúde física e psicológica dos cuidadores e um dos fatores que atuam nesta atenuação são estratégias de enfrentamento voltadas para a emoção e religião (OLIVEIRA NA, et al. 2020).

Nos indivíduos portadores de doenças crônicas como o Diabetes Mellitus, assistidos por uma equipe de atenção primária, é visível, conjuntamente, a percepção de instrumentos como a proximidade familiar e a religiosidade como fatores aliados no enfrentamento dos seus itinerários terapêuticos.

Esses indivíduos visualizam a R/E não somente como fator de fortalecimento das questões referentes diretamente à saúde, mas como fator determinante para toda e quaisquer adversidades de vida ou cotidiano, isso mostra que a R/E interfere em todo o processo saúde-doença (COQUEIRO JM, et al., 2018).

Ainda sob os aspectos da R/E em indivíduos com outras condições de doenças crônicas, também percebemos um coping religioso e espiritual positivo. Em pacientes com insuficiência renal crônica dialítica, foi possível perceber uma correlação positiva de religiosidade intrínseca e organizacional com resiliência. Já nos indivíduos sob cuidados paliativos percebeu-se uma correlação positiva entre resiliência e espiritualidade (ZANDAVALLI RB, et al., 2019).

Quando a associação dos aspectos religiosos é feita com a qualidade de vida relacionada à saúde, em pacientes sob tratamento oncológico, observou-se que o domínio relacionado a religiosidade, espiritualidade e crença desempenhou um recurso de expressão forte nos períodos de confrontação com as adversidades peculiares desta entidade nosológica.

Os aspectos da R/E nos pacientes oncológicos também foi associado a melhor sensação de bem estar, conforto e alívio, tudo isso mesmo diante de um intenso sofrimento ou expectativas em relação à doença (MENEZES, et al., 2018).

Os estudos que fazem a abordagem da R/E no nível primário de atenção à saúde, também trazem evidências que ratificam uma provável associação de hábitos ou estilos de vida danosos ao bem estar, com a pouca ou inexistente espiritualidade. Em relação ao hábito de fumar, intimamente ligado ao desenvolvimento de patologias do aparelho cardiopulmonar, como o câncer de pulmão, a religiosidade pode ser considerado um fator importante na influência deste comportamento. 
A prevalência do tabagismo é maior em indivíduos que consideram não ter religião, seguida por indivíduos que afirmar ter religião, mas não a praticam, que em indivíduos que referem ter religião e praticam tanto intrínseca como extrinsecamente (MARTINEZ EZ, et al., 2019).

É também visualizado, segundo os estudos na APS, uma possível relação de proteção contra o etilismo e a religião, ou seja, as práticas relacionadas à fé como: hábito de frequentar igrejas ou quaisquer encontros religiosos, parece distanciar os indivíduos do uso abusivo de álcool.

Nos indivíduos que referiram ser pertencentes à religião evangélica, percebeu-se maior reprovação moral ao hábito de ingesta alcóolica. Isso pode reforçar as ações das equipes da atenção primária em parceria com representações religiosas da comunidade como atores importantes de reforço positivo no combate aos hábitos de vida não saudáveis (QUEIROZ NR, et al., 2015; MARTINEZ EZ, et al., 2019).

Alguns estudos, trazem os aspectos religiosos como fatores importantes para a saúde mental, e que essa proteção poderia ser explicada pela capacidade da R/E funcionar como um promotor de resiliência, ou seja, na capacidade do indivíduo de superação, de retomada à normalidade, sendo uma forma de coping religioso positivo, onde os aspectos religiosos atuam diretamente como determinante, de forma positiva, para as problemáticas relacionadas à saúde da mente.

Como a APS funciona como a porta de entrada para toda a rede de saúde, e tem um grande potencial de resolutividade, inserir a abordagem religiosa pode impactar nas problemáticas relacionadas à ansiedade, depressão, dentre outras (ZANDAVALLI RB, et al., 2019).

Todavia, outros estudos, que relacionam os aspectos religiosos com a saúde mental, inserem um conceito de total ambivalência relacionada a R/E e a mente, o que pode predizer que, para alguns pacientes com transtornos relacionados à saúde mental, a R/E pode se insinuar como fator negativo ou de piora clínica, 0 que podemos referir como coping religioso negativo. Isso representa que, em se tratando de saúde mental na APS, o impacto da abordagem religiosa pode conferir tanto proteção como possível desamparo ou desajuste clínico (LIAT L, et al., 2014).

Quanto aos indivíduos com déficit cognitivo, mais precisamente pessoas com Doença de Alzheimer, os fatores religiosos podem estar relacionados a trajetória favorável para a função cognitiva. O impacto positivo da religiosidade também é visualizado em indivíduos cuidadores de pessoas com essa patologia, onde toda e qualquer manifestação que dê sentido à vida, representa papel primordial no enfrentamento das dificuldades, a isso se inclui a espiritualidade (TEDRUS GMAS, et al., 2020).

Com relação aos profissionais que compõem a Atenção Primária à saúde, o que podemos perceber, segundo a literatura, sobretudo com o profissional médico, é que a R/E dos pacientes é maior que a dos profissionais quando se compara a crença em Deus, filiação e frequência religiosa.

Existe neste interim uma espécie de paradoxo: por um lado os profissionais reconhecem a importância que os fatores religiosos e espirituais exercem na saúde dos pacientes, porém desconsideram, em parte, o papel de cuidadores desta dimensão, e que esta função deve ser delegada a outros cuidadores.

Um levantamento realizado em 2014 do World Values Survey, apontou que 98,4\% da população brasileira acredita em Deus, em contrapartida cerca de $86,3 \%$ dos médicos brasileiros também pactuam desta crença, número superior ao de médicos nos Estados Unidos que apresentaram percentual de crença em Deus de 68,5\% (AGUIAR PR, et al., 2017; KELLY EP, et al., 2020).

A necessidade de dialogar, ou ser questionado sobre os aspectos religiosos e espirituais é uma necessidade da maioria dos pacientes assistidos pela APS. Muitos percebem um certo distanciamento com os profissionais, o que os impedem de permear pelos campos da religião neste processo.

Estreitar a relação profissional - paciente, promover uma escuta ativa e acolhedora, compreender os aspectos sociais e culturais da população adscrita, bem como exercitar uma clínica ampliada, podem ser caminhos a serem traçados que satisfaçam o desejo do usuário de ser compreendido em sua complexa integralidade, consequente não dissociar os fatores religiosos e espirituais da saúde (DA SILVA MC, et al., 2018; COQUEIRO JM, et al., 2018). 


\section{CONSIDERAÇÕES FINAIS}

É possível perceber, que existe uma sensibilização dos profissionais que compõem este nível de atenção à saúde, no caráter de reconhecer a R/E como fator interferente no processo saúde-doença, todavia, por diversas limitações essa abordagem é negligenciada ou denotada a outros cuidadores. O que as evidências deste estudo encontrou foi que a R/E parece exercer um papel bastante importante no processo saúdedoença dos indivíduos idosos, com doenças crônicas, em relação ao seu itinerário terapêutico e quanto a alguns hábitos como de consumir bebida alcóolica e de fumar, exercendo papel protetivo quanto a estes. Em relação a saúde mental, pode-se visualizar uma ambivalência, ora funcionando como CRE positivo, ora interferindo negativamente no curso clínico, CRE negativo.

\section{REFERÊNCIAS}

1. AGUIAR PR, et al. Religiosidade/Espiritualidade dos Médicos de Família: Avaliação de Alunos da Universidade Aberta do SUS (UNA-SUS). Rev. bras. educ. med., Rio de Janeiro, 2017; 41(02): p. 102-106.

2. ALMEIDA AM, LUCHHETTI G. Panorama das pesquisas em ciência, saúde e espiritualidade. Cien. Cult., São Paulo, 2016; 68, (1): 54-57.

3. COQUEIRO JM, et al. Itinerários terapêuticos de sujeitos com Diabetes Mellitus: linhas fortes e fracas. Revenferm UFPE online, Recife, 2019;12(6):1554-1562.

4. CORREAA CV, et al. Coping religioso/espiritual em processos de saúde e doença: revisão de produção em periódicos brasileiros (2000-2013). RevistPsicoFAE: Pluralidades em Saúde Mental, Curitiba, 2016; 5(1):61-78.

5. DA SILVA MC, et al. Idosos cuidando de idosos: a espiritualidade como alívio das tensões. Rev. Bras. Enferm., Brasília, 2018; 71(5):461-2468.

6. DAMASCENO RF, DA SILVA PLM. Competência cultural na atenção primária: algumas considerações. J ManagPrim Health Care, Sorocaba, 2018; 9:1-8.

7. DE FIGUEIRÊDO RAM, et al. Representações sociais de saúde e doença entre acadêmicos de medicina. Ensino \& Pesquisa, 2020.

8. DE SOUSA MM, et al. A metodologia de revisão integrativa em enfermagem. Revista Investigação em Enfermagem, São Paulo, 2017;21:17-26.

9. DE SOUZA MT, et al. Revisão integrativa: o que é e como fazer. Einstein, São Paulo, 2010; 08(01): 102-106.

10. GIOVANELLA L, et al. Saúde da família: limites e possibilidades para uma abordagem integral de atenção primária à saúde no Brasil.Ciênc. saúde coletiva, Rio de Janeiro, 2009; 14(3): 783-794.

11. INOUE TM, VERCINA MV. Espiritualidade e/ou religiosidade e saúde: uma revisão de literatura. J Health Scilnst, Sorocaba, 2017; 35(2): 127-130.

12. KELLY EP, et al. Does spiritual andreligiousorientationimpacttheclinicalpracticeof healthcare providers? JournalofInterprofessionalCare, 2020; 34,(7): 520-527.

13. LIAT A, et al. Betweenmodernandtraditionalvalues: Informal mental health helpseekingattitudesaccordingtolsraeliArabwomen, primarycarepatientsandtheirproviders. InternationalJournalof Social Psychiatry, 2014; 61(4).

14. LIBSTER M M. Spiritual formation, secularization, andreformof professional nursingandeducation in antebellumAmerica. Journalof Professional Nursing, 2018; 34(1): 47-53.

15. MARTINEZ EZ, et al. Religiosityandpatternsofalcoholconsumptionamongusersofprimary healthcare facilities in Brazil. Cad. saúde colet., Rio de Janeiro, 2019; 27(20):146-157.

16. MARTINEZ EZ, et al. Smoking Prevalence Among Users of Primary Healthcare Units in Brazil: The Role of Religiosity. Journal of Religionand Health, 2017; 56(2).

17. MENEZES RM, et al. Qualidade de vida relacionada à saúde e espiritualidade em pessoas com câncer. Revista Brasileira de Cancerlogia, 2018; 64(1): 9-17.

18. NUNES MGS, et al. Idosos longevos: avaliação da qualidade de vida no domínio da espiritualidade, da religiosidade e de crenças pessoais. Saúde em Debate, Rio de Janeiro, 2017; 41(115): 546-552.

19. OLIVEIRA NA, et al. Elderlycaregiversofotherelderly living withandwithoutchildren: burden, optimismandcopingstrategies. Ciênc. saúde coletiva, Rio de Janeiro, 2020; 25(2): 473-481.

20. PANITZ GO, et al. Instrumentos de abordagem da Espiritualidade na prática clínica. Acta Médica: LIGAS ACADÊMICAS, Rio Grande do Sul, 2018; 39(1): 37-45.

21. QUEIROZ NR, et al. Associação entre o consumo de bebidas alcoólicas e tabaco e a religiosidade. Acta Paulista de Enfermagem, São Paulo, 2015; 28(06): 546-552.

22. TEDRUS GMAS, et al. Religiosidade e qualidade de vida em indivíduos com Doença de Alzheimer e do cuidador: relações com aspectos clínicos e cognitivos. Dementia \& Neuropsychologia, São Paulo, 2020; 14(1): 69-74.

23. TEIXEIRA E, et al. Revisão Integrativa da Literatura passo-a-passo \& convergências com outros métodos de revisão. REUFPI, Teresina, 2013; 02(05): 102-106.

24. VALENTE TCO, et al. Espiritualidade, religiosidade e saúde: velhos debates, novas perspectivas. Interações, Minas Gerais, 2016; 11(20): 85-97.

25. ZANDAVALLI RB, et al. Espiritualidade e resiliência na atenção domiciliar. Revista Brasileira de Medicina de Família e Comunidade, Rio de Janeiro, 2019; 15(42): 13-22. 